\title{
Lista das Nymphaeales de Mato Grosso do Sul, Brasil
}

\author{
Suzana Neves Moreira' \& Vali Joana Pott ${ }^{2}$
}

\begin{abstract}
'Universidade Federal de Minas Gerais, Instituto e Ciências Biológicas, Departamento de Botânica, Avenida Antônio Carlos, 6627, Pampulha, CEP 31270-901, Belo Horizonte, MG, Brasil. suzannanevesmoreira@gmail.com 2Universidade Federal do Mato Grosso do Sul, Instituto e Ciências Biológicas, Laboratório de Botânica, Herbário, CP 549 - CEP 79070-900, Campo Grande, MS, Brasil.
\end{abstract}

\begin{abstract}
RESUMO - Nymphaeales é uma ordem que, no Mato Grosso do Sul, é representada por duas famílias, Nymphaeaceae e Cabombaceae. As informações aqui disponibilizadas foram provenientes de materiais herborizados que estão depositados nos herbários do estado (CGMS e CPAP). São relacionadas 13 espécies (com duas subespécies) das quais 11 pertencem a Nymphaeaceae (Nymphaea amazonum Mart. \& Zucc. subsp. amazonum, N. amazonum subsp. pedersenii Wiersema, N. belophylla Trickett, N. caerulea Savigny, N. gardneriana Planch., N. jamesoniana Planch., N. lingulata Wiersema, N. oxypetala Planch., N. prolifera Wiersema, N. rudgeana G.Mey., Victoria amazonica (Poepp.) J.E.Sowerby) e duas espécies de Cabombaceae (Cabomba furcata Schult. \& Schult. f. e C. haiynesii Wiersema). As duas espécies de Cambombaceae e oito de Nymphaeaceae ocorrem no Pantanal. Nymphaeaceae é a família mais rica, o gênero mais rico, Nymphaea (nove espécies). Nymphaea caerulea é subespontânea, floração diurna. Cabomba furcata tem ampla distribuição e C. haynesii exclusiva no Pantanal.
\end{abstract}

Palavras-chave: Cabombaceae, Nymphaeaceae, florística, Pantanal, taxonomia

\begin{abstract}
Checklist of the Nymphaeales of Mato Grosso do Sul, Brazil. Nymphaeales is an order with two families in Mato Grosso do Sul, Nymphaeaceae and Cabombaceae. This information is based on plant material preserved in the herbaria CGMS and CPAP. We cite 13 species (with two subspecies), 11 belonging to Nymphaeaceae (Nymphaea amazonum Mart. \& Zucc. subsp. amazonum, N. amazonum subsp. pedersenii Wiersema, $N$. belophylla Trickett, $N$. caerulea Savigny, $N$. gardneriana Planch., $N$. jamesoniana Planch., $N$. lingulata Wiersema, $N$. oxypetala Planch., $N$. prolifera Wiersema, N. rudgeana G. Mey., Victoria amazonica (Poepp.) J. E. Sowerby) and two species of Cabombaceae (Cabomba furcata Schul. \& Schult. f. and C. haynesii Wiersema). Eight Nymphaeaceae and both Cabombaceae occur in the Pantanal. The richest family is Nymphaeaceae and the richest genus is Nymphaea (nine species). Nymphaea caerulea is subspontaneous, day flowering. Cabomba furcata is widely distributed and C. haynesii is exclusive to Pantanal.
\end{abstract}

Keywords: Cabombaceae, Nymphaeaceae, floristics, Pantanal, taxonomy

\section{INTRODUÇÃO}

Nymphaeales é uma ordem das Angiospermas basais, claramente monofilética (Hilu et al. 2003) e compreende três famílias, Cabombaceae, Hydatellaceae e Nymphaeaceae. Hydatellaceae ocorre apenas na Austrália e Índia (APG III 2009). Segundo Schneider \& Williamson (1993), a ordem compreende oito gêneros e cerca de 70 espécies de plantas exclusivamente aquáticas. No Brasil, Cabombaceae e Nymphaeaceae são encontradas em todas as regiões (Amaral 2015a).

Nymphaeaceae é representada por ervas aquáticas rizomatosa de folhas simples, flutuantes ou submersas, com lâmina cordada ou peltada (Feres \& Amaral 2003). É considerada uma família relativamente pequena e com seus representantes exclusivamente hidrófilos (Hoehne 1948). A família Nymphaeaceae tem distribuição cosmopolita, possui seis gêneros e 55 espécies (Padgett \& Les 2004), além de numerosos híbridos e variedades (Feres \& Amaral
2003). Nymphaea L. e Victoria Lindl. são os gêneros que ocorrem no Brasil, mas o número de espécies diverge dependendo do autor. Souza \& Lorenzi (2008) citam 10 espécies, enquanto Amaral (2015a) reconhece 19.

Devido à beleza das flores, que são grandes e vistosas, muitas são utilizadas como ornamentais. Alguns representantes exóticos utilizados na decoração de ambientes passaram a ocorrer de maneira subespontânea (Souza \& Lorenzi 2008). Victoria amazonica, com sua beleza cênica, ocorre nas enseadas e meandros abandonados do Rio Paraguai. Para o Brasil o principal tratamento taxonômico sobre a família é o de Caspary (1878) na Flora Brasiliensis e para o Pantanal o de Pott (1998).

Cabombaceae é representada por ervas aquáticas submersas fixas com folhas palmatissectas; as folhas flutuantes são peltadas ou sagitadas (Cook 2004) durante a floração, ou ausentes (Amaral et al. 2008). As folhas flutuantes servem para a sustentação das flores emergentes. Possui distribuição cosmopolita e é representada mundialmente por dois gêneros 
(Brasenia Schreb. e Cabomba Aubl.) e aproximadamente cinco espécies, no Brasil é representada apenas por Cabomba. São espécies de habitat exclusivamente aquático, com flores emersas e vistosas (Souza \& Lorenzi 2008). Assim como para Nymphaeaceae, o principal tratamento taxonômico sobre a família Cabombaceae é o de Caspary (1878) na Flora Brasiliensis.

\section{Principais Grupos de Pesquisa e Lacunas de Conhecimento}

Existe trabalho de referência desenvolvido por Pott (1998), onde apresentou uma listagem das espécies de Nymphaeaceae que ocorrem no Pantanal de Mato Grosso do Sul e Mato Grosso e um guia de identificação de plantas aquáticas do Pantanal de Pott \& Pott (2000). A partir destes trabalhos, não houve incremento no número de espécies à lista florística do estado, excetuando o trabalho de Amador et al. (2013), onde cita uma nova ocorrência de Nymphaea para o Mato Grosso do Sul.

Trabalhos específicos vêm sendo realizados em diferentes estados brasileiros, como o estudo de Sousa \& Matias (2013) no Ceará, onde inventariaram a flora de Nymphaeaceae e por Lima et al. (2012) para a Bahia onde inventariaram Cabombaceae. A Lista da Flora do Brasil para Nymphaceae e Cabombaceae é liderada por Amaral, M.C.E.; Pellegrini, M.O.O.; Lima, C.T., sendo a última atualização em 2015.

Com o Projeto Biota MS, haverá mais expedições em áreas pouco coletadas no estado, como é o caso das nascentes da bacia do Miranda e os resultados podem ser ampliados ou mesmo corroborados com o que está disponível até o presente momento.

\section{Principais Acervos e Perspectivas de Pesquisa para o Grupo nos próximos 10 anos}

Os principais Herbários que possuem representantes de Nymphaeales são: Herbário CGMS, CPAP, COR e DDMS, siglas de acordo com Thiers (2015). Com o projeto Biota MS, é provável que haja acréscimos à lista das espécies para as demais famílias, contudo, acreditamos que a possibilidade de incremento de espécies para Nymphaeaceae e Cabombaceae seja pequena, exceto em caso de alguma espécie ser introduzida por se tratar de um grupo de plantas ornamentais.

\section{MATERIAL E MÉTODOS}

A presente lista de Nymphaeales apresenta as espécies citadas nas publicações sobre a flora do Mato Grosso do Sul, Pott \& Pott (1997), Dubs (1998), Pott (1998), Pott \& Pott (1999), Scremin-Dias (1999), Pott \& Pott (2000). Destes, Dubs (1998) é a obra mais importante, que lista as espécies de MS e MT compiladas do herbário MBM e de vários herbários europeus como Z, S, K, E. Além destes, foram adicionados representantes dos espécimes depositados em Herbários do estado, cada espécie com um voucher correspondente. A Lista da Flora do Brasil (Forzza et al. 2015) foi utilizada como plataforma de referência para validar os nomes das espécies.

\section{RESULTADOS E DISCUSSÃO}

Foram inventariadas 13 espécies de Nymphaeales no estado do Mato Grosso do Sul (Quadro 1), sendo 10 espécies de Nymphaeaceae, com duas subespécies, e duas espécies de Cabombaceae. A família com maior número de espécies é Nymphaeaceae, sendo que o gênero com maior número de espécies é Nymphaea (nove espécies) que somam cerca de $75 \%$ do total.

\section{Nymphaeaceae}

No mundo são reportadas 60 espécies de Nymphaeaceae (Bosch et al. 2008) e para o Brasil, aproximadamente 15 (Souza \& Lorenzi 2012). Dubs (1998) em seu trabalho Prodromus Florae Matogrossensis cita cinco espécies para o estado, Nymphaea amazonum subsp. perdersenii, $N$. gardneriana, $N$. jamesoniana, $N$. oxypetala e Victoria cruziana (sin. Victoria amazonica).Das espécies listadas, apenas duas ocorrem fora do Pantanal, Nymphaea caerulea e N. rudgeana. Nymphaea caerulea é nativa da África do Sul ocorre como subespontânea no Centro Oeste e Sudeste do Brasil (Amaral et al. 2008). Segundo Wiersema (1987), todas as espécies, com exceção de $N$. caerulea apresentam floração noturna. Das 19 espécies citadas por Amaral (2015a) na Lista da Flora do Brasil, nove ocorrem no Mato Grosso do Sul, contudo, apenas duas foram citadas para o estado, Nymphaea jamesoniana e $N$. oxypetala, necessitando a atualização da referia plataforma. Em Dubs (1998) são citadas cinco espécies da atual lista, sendo que Victoria amazonica foi citada como V. cruziana D’Orbigny (sinônimo). Na lista das Espermatófitas do estado de São Paulo (Wanderley et al. 2011), foram citadas sete espécies, destas, quatro são comuns às encontradas até o momento no Mato Grosso do Sul (Nymphaea amazonum, $N$. caerulea, $N$. gardneriana e $N$. rudgeana). Para o Rio Grande do Sul foram citadas 3 espécies por Irgang \& Gastal (1996), N. amazonum, N. prolifera e uma não identificada. Em Bove \& Paz (2009), para as restingas de Jurubatiba, RJ, são citadas Nymphaea pulchella DC. e N. rudgeana. Nymphaea rudgeana no MS foi encontrada apenas no Rio Sucuriú e PCH Alto Sucuriú em Costa Rica. Sousa \& Matias (2013) citaram sete espécies para o Ceará, $N$. amazonum, $N$. lasiophylla, $N$. jamesoniana, $N$. lingulata, $N$. pulchella, $N$. rudgeana e $N$. tenerinervia, sendo $N$. lasiophylla a mais comum, contudo, apenas três destas ocorrem no Mato Grosso do sul. Nymphaeae belophylla foi citada pela primeira vez para o Mato Grosso do Sul por Amador et al. (2013).

\section{Cabombaceae}

No mundo existem cerca de cinco espécies e no Brasil são estimadas de três a quatro espécies (Souza \& Lorenzi 2012). Segundo Matias et al. (2012), podem ser encontrados representantes em todos os domínios fitogeográficos brasileiros, Amazônia, Caatinga, Cerrado, Mata Atlântica, Pampa e Pantanal. Dubs (1998) cita duas espécies para Mato Grosso do Sul: Cabomba 
Quadro 1. Lista das espécies de Nymphaeales (Cabombaceae e Nymphaeaceae) já citadas e/ou coletadas no estado de Mato Grosso do Sul, relacionadas por família, segundo APG III (2009). (*) espécie introduzida.

\begin{tabular}{|c|c|}
\hline Família/Espécies & Voucher (Herbário) \\
\hline \multicolumn{2}{|l|}{ Cabombaceae } \\
\hline Cabomba furcata Schult. \& Schult. f. & S.N. Moreira 302 (CGMS) \\
\hline C. haynesii Wiersema & V.J. Pott 1782 (CGMS) \\
\hline \multicolumn{2}{|l|}{ Nymphaeaceae } \\
\hline Nymphaea amazonum subsp. amazonum Mart. \& Zucc. & V.J. Pott 6348 (CGMS) \\
\hline N. amazonum subsp. pedersenii Wiersema & V.J. Pott 3365 (CPAP/CGMS) \\
\hline N. belophylla Trickett & G. Amador 235 (CGMS) \\
\hline N. caerulea Savigny (*) & V.J. Pott 2868 (CPAP/CGMS) \\
\hline N. gardneriana Planch. & V.J. Pott 3888 (CPAP/CGMS) \\
\hline N. jamesoniana Planch. & V.J. Pott 4725 (CGMS) \\
\hline N. lingulata Wiersema & V.J. Pott 3282 (CPAP/CGMS) \\
\hline N. oxypetala Planch. & V.J. Pott 3863 (CPAP/CGMS) \\
\hline N. prolifera Wiersema & V.J. Pott 6350 (CGMS) \\
\hline N. rudgeana G.Mey. & V.J. Pott \& A. Pott 9178 (CGMS) \\
\hline Victoria amazonica (Poepp.) J.E.Sowerby & V.J. Pott 1999 (CPAP/CGMS) \\
\hline
\end{tabular}

furcata (sinônimo C. piauhiensis Gardner) e C. haynesii. Cabomba haynesii ocorre somente nos estados de Mato Grosso do Sul (na rub-região do Nabileque, sul do Pantanal) e Espírito Santo, de acordo com Amaral (2015b) na Lista da Flora do Brasil. Provavelmente ocorra em outras regiões circunvizinhas, como Minas Gerais e Goiás, já no estado de São Paulo onde, teoricamente, existe a possibilidade de ser encontrada, não foi listada no trabalho desenvolvido por Wanderley et al. (2011). Ainda, segundo Wanderley et al. (2011), excetuando o Sul do Brasil, podemos encontrar C. furcata em todas as regiões do País.

As espécies que ocorrem no MS podem ser distinguidas de acordo com o número de folhas e cor das flores: $C$. furcata apresenta três folhas por verticilo e flores arroxeadas e $C$. haynesii apenas duas por verticilo e flores esbranquiçadas.

Nymphaeaceae e Cabombaceae são famílias relativamente pequenas e acreditamos que, considerando os esforços de coleta até o presente momento no estado, a presente lista contemple a diversidade de suas espécies. Dubs (1998), Pott \& Pott (1999) e Pott \& Pott (2000) citam a maioria das espécies encontradas no Mato Grosso do Sul, assim como a lista das espécies da Flora do Brasil (Amaral 2015 a,b).

\section{AGRADECIMENTOS}

À Coordenação de Aperfeiçoamento de Pessoal de Ensino Superior pela bolsa de Doutorado concedida a primeira autora e à Ana Cristina de Meira Cristaldo, bolsista Biota MS, pelo auxílio no Herbário CGMS.

\section{REFERÊNCIAS}

Amador, G. A., Damasceno-Júnior, G. A., Silva, R. H., Pott, A. \& Pott, V. J. 2013. Nymphaeaceae, Nymphaea belophylla Trickett: New state record. Check List 9(2): 440-442.

Amaral, M.C.E. 2015a. Nymphaeaceae. In Lista de Espécies da Flora do Brasil. Jardim Botânico do Rio de Janeiro. Disponível em: http://
floradobrasil.jbrj.gov.br/2012/FB000173. Acessado em 10.09.2015. 2015b. Cabombaceae. In Lista de Espécies da Flora do Brasil. Jardim Botânico do Rio de Janeiro. Disponível em: http:// floradobrasil.jbrj.gov.br/2012/FB034569. Acessado em 10.09.2015. Amaral, M.C.E., Bittrich, V., Faria, A.D., Anderson, L.O. \& Aona, L.Y.S. 2008. Guia de Campo para plantas aquáticas e palustres do estado de São Paulo. Holos Editora, Ribeirão Preto. 452p.

Angiosperm Phylogeny Group - APG (2009). An update of the Angiosperm Phylogeny Group classification for the orders and families of flowering plants: APG III. The Linnean Society of London. Botanical Journal of the Linnean Society 161:105-121.

Bosch, T., Löhne, C. \& Wiersema, J.H. 2008. Phylogeny and evolutionary patterns in Nymphaeales: integrating genes, genomes and morphology. Taxon 57: 1052-1081.

Bove, C. P.; Paz, J. 2009. Guia de Campo das Plantas Aquáticas do Parque Nacional da Restinga de Jurubatiba. Editora do Museu Nacional, Rio de Janeiro. $176 \mathrm{p}$.

Caspary, R. 1878. Nymphaeaceae. In Flora Brasiliensis (C.F.P. Martius, A.W.Eichler \& I. Urban, eds.). Frif. Freischer, Lipsiae, v. 4, p.131-184.

Cook, C.D.K. 2004. Aquatic and wetland Plants of Southern Africa. Backhuys Publishers, Leiden The Netherlands. 281 p.

Dubs, B. 1998. Prodromus Florae Matogrossensis. Betrona Verlag, Küsnacht. 444 p.

Feres, F. \& Amaral, M.C.E. 2003. Nymphaeaceae. In Flora Fanerogâmica do Estado de São Paulo (M.G.L. Wanderley, G.J. Shepherd, A.M. Giulietti \& T.S. Melhen, eds.). Fundação de Amparo à Pesquisa de São Paulo, RiMa. São Paulo, v. 3, p. 241-245.

Forzza et al. 2015. Lista de Espécies da Flora do Brasil. Jardim Botânico do Rio de Janeiro. Disponível em: http://floradobrasil.jbrj.gov.br/. Acessado em: 10.09.2015.

Hilu, K. W., T. Borsch, K. Müller, D. E. Soltis, P. S. Soltis, V. Savolainen, M. W. Chase, M. P.

Powell, L. A. Alice, R. Evans, H. Sauquet, C. Neinhuis, T. A. B. Slotta, J. G. Rohwer, C. S. Campbell, \& L. W. Chatrou (2003). Angiosperm phylogeny based on matK sequence information. American Journal of Botany 90: 1758-1776.

Hoehne, F.C. 1948. Plantas aquáticas. Instituto de Botânica, São Paulo. $168 \mathrm{p}$.

Irgang, B.E. \& Gastal JR., C.V.S. 1996. Plantas aquáticas da planície costeira do Rio Grande do Sul. Porto Alegre. 290p.

Lima, C.T., Giulietti, A.M. \& Santos, F.A.R. 2012. Flora da Bahia: Cabombaceae. Sitientibus. Série Ciências Biológicas 12: 61-68.

Padgett, D.J. \& Les, D.H. 2004. Nymphaeaceae. In Flowering plants of the Neotropics (N. Smith, S.A. Mori, A. Henderson, D.W. Stevenson, \& S. V. Heald, eds.). Princeton University Press, Princeton, p. 271-273.

Pott, V.J. 1998. A família Nymphaeaceae no Pantanal, MS, MT, Brasil. Acta Botanica Brasilica 12(2):183-194. 
Pott, V.J. \& Pott, A. 1997. Checklist das macrófitas aquáticas do Pantanal, Brasil. Acta Botanica Brasilica 11(2): 215-227.

1999. Flora do Pantanal - listagem atual de fanerógamas. In Anais do Simpósio sobre Recursos Naturais e Sócio-Econômicos do Pantanal, Manejo e Conservação, Corumbá, Embrapa Pantanal. p. 297-325.

2000. Plantas aquáticas do Pantanal. Empresa Brasileira de Pesquisa Agropecuária, Corumbá. 353p.

Schneider, E.L \& Williamson, P.S. 1993. Nymphaeaceae. In Families and genera of flowering plants (K. Kubitzki, ed.). Springer, Berlin. p. 486-493.

Scremin-Dias, E. 1999. O retorno à origem aquática. In Nos Jardins Submersos da Bodoquena: guia para identificação de plantas aquáticas de Bonito (E. Dias, V.J.Pott \& P.R. Souza, eds). Editora da Universidade Federal de Mato Grosso do Sul, Campo Grande, p. $25-41$.

Sousa, D.J.L. \& Matias, L.Q. 2013. A família Nymphaeaceae no estado do Ceará, Brasil. Rodriguésia 64(1): 49-59.

Souza, V.C. \& Lorenzi, H. 2008. Botânica Sistemática (Guia Ilustrado para identificação das famílias Fanerogâmicas nativas e exóticas no Brasil, baseado em APGII). Instituto Plantarum de Estudos da Flora, Nova Odessa, São Paulo. 640p.

2012. Botânica Sistemática - Guia Ilustrado para identificação das famílias Fanerogâmicas nativas e exóticas no Brasil, baseado em APGII. Instituto Plantarum de Estudos da Flora, Nova Odessa, São Paulo. 768p.

Thiers, B. 2012. Index Herbariorum: A global directory of public herbaria and associated staff. New York Botanical Garden's Virtual Herbarium. http://sweetgum.nybg.org/ih/. Acessado em 10.09. 2015.

Wanderley, M.G.L.; Shepherd, G.J.; Martins, S.E.; Estrada, T.E.M.D.; Romanini, R.P.; Koch, I.; Pirani, J.R.; Melhem, T.S.; Harley, A.M.G.; Kinoshita, L.S.; Magenta, M.A.G.; Wagner, H.M.L.; Barros, F.; Lohmann, L.G.; Amaral, M.C.E.; Cordeiro, I.; Aragaki, S.; Bianchini, R.S. \& Esteves, G.L. 2011. Checklist das Spermatophyta do Checklist das Spermatophyta do Estado de São Paulo, Brasil. Biota Neotropica 11: $193-390$.

Wiersema, J. H. 1987. A monograph of Nymphaea subgen. Hydrocallis (Nymphaeaceae). Systematic Botany Monography 16: 1-112. 